\title{
Impact of reformed RM Encoded data on PAPR of Multicode CDMA under MSK
}

\author{
* S.Rizwan-ul-Hasan, ** Najeeb Siddiqui and *** Shakil Ahmed
}

\begin{abstract}
In this research work, the effect of Reformed Reed Muller Encoded Data (RRMED) on Peak to Average Power Ratio (PAPR) of Multicode Code Division Multiple Access (MC-CDMA) systems was studied under Minimum Shift Keying (MSK) modulation. Simulation studies revealed that implementation of RRMED on PAPR with MSK modulation can save up to $7 \mathrm{~dB}$ of transmitter power compared to the un-coded PAPR of MC-CDMA systems.

Simulation results further proved that MSK modulation also plays an important role in reducing PAPR as compared to binary Phase Shift Keying (PSK).
\end{abstract}

\section{Index Terms -MC-CDMA, MSK, PAPR, RM}

\section{INTRODUCTION}

MC-CDMA is a multiple access technique, which include backward compatibility, simplicity, high data rates, error correcting capability and orthogonality. The concept behind MC-CDMA is to allocate several channels to a user who wants to relay at a desired data rates $[1,7]$. These several channels present at the base station as several users. Each channel is then multiplied by the standard data rates, which boost up standard data rates $[1,2,3,8]$.

MC-CDMA depends on addition of $\mathrm{n}$ channels of standard data rates for transmission, from an orthogonal square matrix known as discrete WHT. This addition of $\mathrm{n}$ signals yields in high amplitude, which is $\mathrm{n}$ times the mean signal power. Normally, $\mathrm{n}=2^{\mathrm{m}}$, where $\mathrm{m}$ is within limit of 2 and 6 [1-3]. Thus, transmission of MC-CDMA signals with no malformation requires a pricy power amplifier (PA), which should work in its linear region.

Because, MC-CDMA suffers from high peaks, which consequently degrades performance of the (PA) at the forward link and we know that PA works efficiently in its linear area. Hence, a procedure may be formulated for calculating the linearity of the PA $[1,2,3,9,10]$.

A technique to gauge of high amplitude is known as PAPR - the amount of maximum power that a PA could generate versus its mean power $[10,11]$. In order to maintain the linearity of PA, its Input Back Off (IBO) needs to be high.

\section{A. Related work and Motivation}

There are several methods to reduce PAPR $[1,3,7,9,12,21]$. But, all of these methods to reduce PAPR are under binary PSK, 16-QAM, quaternary or octary signaling modulation. There are several advantages of MSK over the said modulation scheme, like spectral efficiency, constant envelope and low PAPR.

This provides obvious motivation to use MSK modulation, instead of binary PSK with RRMED sequence to evaluate PAPR of MCCDMA [18].

\section{B. Contribution and outline}

In the next section-II we will introduce background. Section-III contains proposed methodology, which includes contribution. The main contribution of section-IV is simulation, which proves that MSK further reduces PAPR and section- $\mathrm{V}$ states conclusion and open issues.

\section{BACKGROUND}

\section{A. Reed Muller codes}

Reed Muller codes or RM codes are oldest, finest error correcting and easy to understand codes [19]. They are useful in telecommunication. The distinguish feature of RM codes is that they are easy to decrypt using majority logic circuits [22]. RM code may be defined as $R M(r, m)$ of order $r$, where $r=0$, 1 ....m; has the set of three parameters (n, k, d) as follows:-

$$
\left[n=2^{m}, k=\sum_{i=0}^{r}\left(\begin{array}{l}
m \\
i
\end{array}\right), d=2^{m-r}\right]
$$

Where $\mathrm{m}$ is varying, $\mathrm{n}$ denotes the size of the data, $\mathrm{k}$ is used to find out the number of rows in RM code and $d$ is the minimum distance. Large order RM code can iteratively be formed from the lower order. They are often used as to construct different codes.

\footnotetext{
* Department of Computer Engineering, Sir Syed University of Engineering \& Technology, Karachi, Pakistan. srhasan@ ssuet.edu.pk
} 


\section{B. Walsh Hadamard Transform (WHTn)}

WHTn of dimension $\mathrm{n}$ by $\mathrm{n}$ is a discrete orthogonal transform of matrix; $2 \mathrm{~m} \times 2 \mathrm{~m}$. Recursion is used to generate higher order matrices from the lower order as follows:-

$\operatorname{WHT}\left(2^{m}\right)=\left[\begin{array}{l}\text { WHT }\left(2^{m-1}\right) W H T\left(2^{m-1}\right) \\ \operatorname{WHT}\left(2^{m-1}\right) \operatorname{WHT}\left(2^{m-1}\right)\end{array}\right]$,

WHT $(1)=1$

\section{PAPR}

Now we define PAPR as follows: - Let Di represents an $i^{\text {th }}$. encoded data (with RRMED), then PAPR can mathematically be (with the corresponding transmitted signal $\mathrm{SDi}(\mathrm{t})$ ) stated as follows:

$$
\operatorname{PAPR}(D i)=(1 / n) \max |D i \times S D i(t)|^{2}
$$

Where, PAPR(Di) is the peak power of encoded data Di and $\mathrm{n}$ is length of WHT. For an uncoded data the maximum PAPR is equal to $n$ (number of channels) and the output of WHT is also various level of signal varying from $-n$ to $+n$.For uncoded data the maximum PAPR is equal to $n$ [3]. The lower and upper problem was resolved by reducing the phase jump from 180 degree to 90 degree in Offset Quadrature Phase Shift Keying (OPQSK). But 90 degree phase difference was still there in OPQSK compare to Quadrature Phase Shift Keying (QPSK). This short phase jump problem of OQPSK was further rectified and the resultant modulation scheme is known as continuous phase modulation (CPM). Minimum shift Keying (MSK) is one of the scheme [23]. In MSK there are no phase discontinuities [24]. Since there is no phase discontinuities in MSK signal, therefore it shows reduced power spectrum than Binary Phase-Shift Keying (BPSK) [25].

In OQPSK, the rectangular shape pulses are used. If these rectangular shape pulses are replaced by sinusoidal pulse shapes i.e. by $\operatorname{Cos}(\pi / 2 \mathrm{~T})$ and $\operatorname{Sin}(\pi / 2 \mathrm{~T})$ for the in-phase (I) and quadrature (Q) component respectively. This is also known as MSK [26]. MSK provides constant envelope and low PAPR. Its application ranges from Global System for Mobile Communication (GSM) to micro satellite communication, positioning and navigation system, hybrid optical / wireless communication system, and deep space communication [27].

\section{PROPOSED METHODOLOGY}

We would like to propose Minimum Shift Keying (MSK) to add to the above contributions as mentioned in motivation and related work. Our communication model is depicted in Fig. 1, which consists of data ' $D$ ', data encoder with RRMED, MSK (for spectral efficiency and low power), WHT and finally signal, $\mathrm{SDi}(\mathrm{t})$ is relayed to the receiver over the wireless interface. At the receiver end inverse transformation and decrypting is performed to recover the un-coded data ' $D$ '.

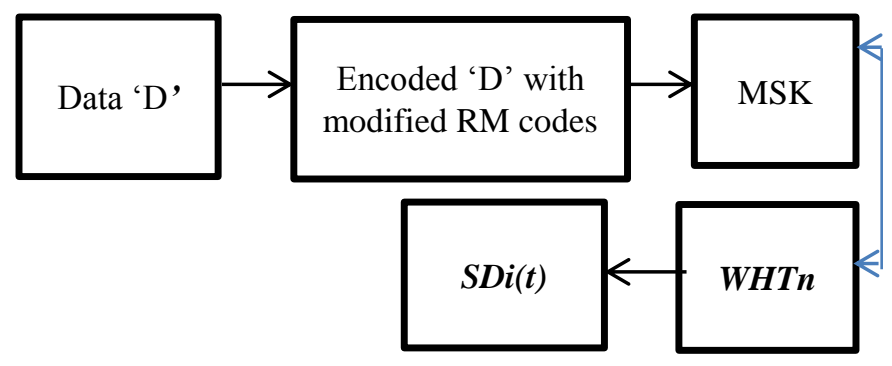

Fig. 1. MC-CDMA transmitter

\section{SIMULATION}

\section{A. Discussion}

Table I shows simulation parameters we used to design the MC-CDMA transmitter. We start our discussion first for effect of RRMED on PAPR under MSK modulation and second PAPR for MSK vs. PSK.

For RM code and un-coded message length, we produce 10,000 random data segments. As shown in Fig. 1, Encoded data ' $\mathrm{D}$ ' with RRMED is modulated under MSK. The modulated data is transformed into orthogonal signal using WHT. The resultant PAPR is constant and reduced. But for un-coded data, PAPR is variable and high.

TABLE I

Simulation parameters

\begin{tabular}{|c|}
\hline Simulation tool : MATLAB \\
\hline Multi Access Technique : MC-CDMA \\
\hline WHT (n x n) : n=4,8,16,32 and 64 \\
\hline Modulation: MSK \\
\hline Channel Coding : RM \\
$(0,2) ;(0,3) ;(0,4) ;(0,5) ;(0,6) ;(1,2) ;(1,3) ;(1,4) ;(1,5) ;(1,6) ;$ \\
$(2,2),(2,3),(2,4),(2,5)$ and 2,6$)$ \\
\hline Random data blocks : 10,000 for each message length of \\
$3,4,5,6,7$, and 8 \\
corresponding to RM codes $(1,2) ;(1,3) ;(1,4) ;(1,5) ;(1,6) ;(2,3)$ \\
\hline
\end{tabular}

Table II, shows the comparison of maximum PAPR under MSK for RM codes of order 0,1 st and 2nd. All of the said orders has constant and low PAPR, except $\operatorname{RM}(2,4), \operatorname{RM}(2,5)$ and $\operatorname{RM}(2,6)$, which has low PAPR compare to original PAPR, but not constant. Verification of constant PAPR, only shown in figures 3 and 4 for $\operatorname{RM}(1,6)$ and $\operatorname{RM}(2,3)$. Table II and Fig. 2, 3 and 4 also demonstrates that RRMED with MSK modulation provides minimum and unvarying PAPR. In some cases PAPR is much lower for e.g. $\operatorname{RM}(0.2), \operatorname{RM}(0,3)$, $\mathrm{RM}(04), \mathrm{RM}(0,5), \mathrm{RM}(1,2), \mathrm{RM}(1,3)$, and $\mathrm{RM}(1,5)$ as shown in figures 2 and 3 respectively. 
TABLE II

Maximum PAPR (dB) for coded (with modified RM codes) and uncoded data under MSK modulation

\begin{tabular}{|c|c|c|c|c|c|c|c|c|c|}
\hline \multicolumn{2}{|c|}{ WHT $(4 \times 4)$} & \multicolumn{2}{|c|}{ WHT $(8 \times 8)$} & \multicolumn{2}{|c|}{ WHT (16x16) } & \multicolumn{2}{|c|}{ WHT $(32 \times 32)$} & \multicolumn{2}{|c|}{ WHT $(64 \times 64)$} \\
\hline \multicolumn{10}{|c|}{ Zero Order Reed-Muller Codes } \\
\hline original & $\mathrm{RM}(0,2)$ & original & $\mathrm{RM}(0,3)$ & original & $\mathrm{RM}(0,4)$ & original & $\mathrm{RM}(0,5)$ & original & $\mathrm{RM}(0,6)$ \\
\hline 3 & 0 & 6 & 0 & 7 & 0 & 10 & 6 & 9.6 & 9 \\
\hline \multicolumn{10}{|c|}{ First Order Reed-Muller Codes } \\
\hline original & $\mathrm{RM}(1,2)$ & original & $\mathrm{RM}(1,3)$ & original & $\mathrm{RM}(1,4)$ & original & $\mathrm{RM}(1,5)$ & original & $\mathrm{RM}(1,6)$ \\
\hline 3 & 0 & 6 & 0 & 9 & 6.5 & 9.7 & 6 & 11.5 & 9 \\
\hline \multicolumn{10}{|c|}{ Second Order Reed-Muller Codes } \\
\hline original & $\mathrm{RM}(2,2)$ & original & $\mathrm{RM}(2,3)$ & original & $\mathrm{RM}(2,4)$ & original & $\mathrm{RM}(2,5)$ & original & $\mathrm{RM}(2,6)$ \\
\hline 3 & 3 & 6 & 4 & 8 & 6.5 & 9.67 & 7 & 10.23 & 9 \\
\hline
\end{tabular}

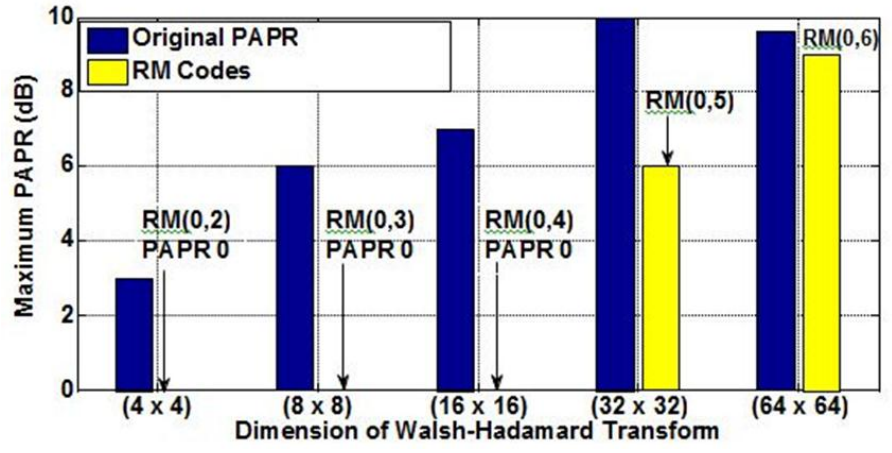

Fig. 2. PAPR Original vs. Coded for RM zero order

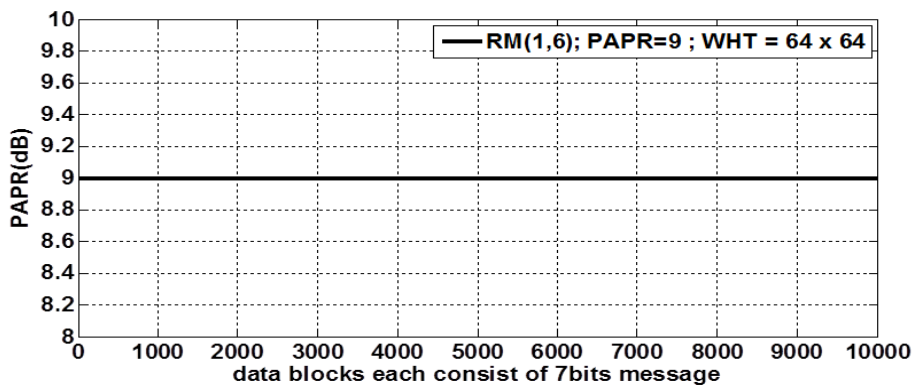

Fig. 3. Constant PAPR for RM(1,6)

Table III

PAPR (dB) for MSK vs. PSK

\begin{tabular}{|c|l|c|c|}
\hline WHT & RMC & MSK & PSK \\
\hline $4 \times 4$ & $\mathrm{RM}(1,2)$ & 0 & 0 \\
\hline $8 \times 8$ & $\mathrm{RM}(1,3)$ & 0 & 3 \\
\hline $16 \times 16$ & $\mathrm{RM}(1,4)$ & 6.5 & 9.5 \\
\hline $32 \times 32$ & $\mathrm{RM}(1,5)$ & 6 & 9 \\
\hline $64 \times 64$ & $\mathrm{RM}(1,6)$ & 9 & 12 \\
\hline $8 \times 8$ & $\mathrm{RM}(2,3)$ & 4 & 6.5 \\
\hline $8 \times 8$ & $\mathrm{RM}(2,4)$ & 6.5 & 6 \\
\hline $8 \times 8$ & $\mathrm{RM}(2,5)$ & 7 & 9 \\
\hline $8 \times 8$ & $\mathrm{RM}(2,6)$ & 9 & 12 \\
\hline
\end{tabular}




\section{REFERENCES}

[1] K. G. Paterson, "On codes with low peak-to-average power ratio for multicode CDMA," IEEE Trans. Inf. Theory, vol. 50, no. 3, pp. 550-559, March 2004.

[2] K. G. Paterson, "Sequences for OFDM and multi-codes CDMA: Two problems in algebraic coding theory," in SETA 2001 (Discrete Mathematics and Theortical Computer Science Series), Berlin, 2002, pp. 46-71.

[3] Kai Uwe Schmidt, "On Spectrally Bounded Codes For Multicarrier Communications," Vogt Verlag, 2007.

[4] Cheol Yong Ahn, Chi Hun Ahn, Seung Moon Ryu, and Dong Ku Kim, "Performance of the Multi Phased MC-CDMA System with Non Linear Amplifier," in Wireless Communications and Networking Conference, vol. 1, 2002.

[5] R. Prasad and S. Hara, "An Overview of Multi-Carrier CDMA," in Spread Spectrum Techniques and Application proceedings, vol. 1, Mainz, 1996, pp. 107-114.

[6] A. C. McCormick and E. A. Al-Susa, "Multi-Carrier CDMA for future Generation Mobile Communication," Electronics \& Communication Engineering Journal, vol. 14, pp. 52-60, April 2002.

[7] Tony.Ottosson, "Precoding in Multicode DS-CDMA System," in IEEE International Symposium on Information Theory, 1997, p. 351

[8] Kai Uwe Schmidt, "Quaternary Constant-Amplitude Codes for multicode CDMA ," IEEE Transaction Information Theory, vol. 55, no. 4, pp. 1824-1832, April 2009.

[9] K. Sathananthan and Tellambura, "Peak-to-Average Power Ratio Analysis in Multicode CDMA," in Vehicular Technology Conference, vol. 1, 2002.

[10] A. Behravan and T. Erikson, "PAPR as measure for OFDM systems with non linearity," 5th International Symposium on Wireless Personal Multimedia Communiactions, vol. 1, pp. 149153, october 2002.

[11] Syed Rizwanul Hasan, Shakil Ahmed, and Mumtazul Imam, "PAPR as measure for OFDM with non-linearity," in Proc.7th CIIT workshop on Research Computing, Lahore, 2008, pp. 114117.

[12] Syed Rizwan-ul-Hasan, "Effect of using RM code to control PAPR in MCCDMA and OFDM," in 14th. IEEE International Multitopic Conference 2011, Karachi, 2011, pp. 329-333.

[13] Henrik Schulze and Christian Luders, Theory and application of OFDM and CDMA Widweband Wireless Communications.: John Wiley and Sons. 1td, 2005.

[14] Nam Yul Yu, "A Theortical study of Peak-to-Average Power Ratio in Reed-Muller Coded Multicarriar CDMA," in Information Sciences and Systems(CISS) 44 th annual conferences, Princeton, 2010, pp. 1-6.
[15] Seung Hee Han and Jae Hong Lee, "An Overview of Peak-toAverage Power ratio reduction techniques for Multicarrier Transmission," in Wireless Communications, vol. 12, April 2005, pp. 56-65.

[16] R. N. Braithwaite, "Using Walsh Code Selection To Reduce the Power Variance of Band-Limited Forward Link CDMA waveforms," Selected areas in Communications, IEEE Journal, vol. 18 , no. 11 , pp. 2260-2269, November 2000.

[17] Cheongju,Tran Phuong Hoa,Kang Mi Lee,Sang-Woo Kim,Jin-Soo Park Heung-Gyoon Rya, "Improvement of the Power efficiency of HPA by the PAPR reduction and predistortion," Consumer Electronics,IEEE Transaction, vol. 50, no. 1, pp. 119-124, 2004.

[18] Yang Zhang, Qiang Ni, Hsiao-Hwa Chen, and Yonghua Song, "An intelligent Genetic Alogorithm for PAPR reduction in MultiCarriar CDMA Wireless Systems," in Wireless Communication \& Mobile Computing Conference, 2008, pp. 1052-1057.

[19] S. Litson, Peak power control in Multi-Carrier Communications.: Cambridge University press, 2007.

[20] Dongsu Kim,Junghwan Son, Yunsung Cho,Jooseung Kim,Byungjoon Park,Bumman Kim Jungjoon Kim, "Push the Envelope," IEEE Microwave Magazine, pp. 68-81, 2013.

[21] S. R. Hasan and N. Siddiqui, "Algorithm for reducing PAPR of Multi-code CDMA using Reed Muller codes," IET Eectronics Letters, vol. 52, no. 11, pp. 926-928, May 2016.

[22] F. J. McWilliams and N. J.A. Sloane, The Theory of ErrorsCorrting Codes. North Holland, 2006, vol. 16.

[23] Bernard Skalar, Digital Communications: Fundamentals and Applications, 2nd ed.: Pearson Education, 2006.

[24] Ian Poole. What is MSK, Minimum Space Keying Modulation. [Online]. http://www.radio-electronics.com/info/rf-technologydesign

[25] Li Tao et al., "A Modulation scheme for $100 \mathrm{~Gb} / \mathrm{s}$ modified minimum-shift keying format based on imblanced bias in IQ components," Optical Fiber Technology, vol. 17, no. 6, pp. 601607, 2011.

[26] Krishina Sankar. (2009, June 16) MSK transmitter and receiver. [Online]. http://www.dsplog.com/2009/06/16/msk-transmitterreceiver/

[27] Adionel Guimar Dayan, "Contributions to the Understanding of the MSK modulation," Revista Telecommunicacoes, vol. 11, no. 01, 2008.

[28] Sabastian Raaphorst, "Reed-Muller Codes," Carleton University, May 9, 2003.

[29] Ben Cooke, "Reed Muller Error Correcting Codes," MIT Undergraduate Journal of Mathematics, pp. 21-27, 1999. 